\title{
Correction to: An Itō Formula in the Space of Tempered Distributions
}

\section{Suprio Bhar ${ }^{1}$}

\section{Correction to: J Theor Probab (2017) 30:510-528 DOI 10.1007/s10959-015-0639-3}

The following corrections are required in Theorem 4.7 of [2].

Recall that the following condition is included in the assumptions (see [1, Chapt. 6, Sect. 2]) made on the coefficient $\bar{F}$; for example, there exists $K>0$ such that

$$
\int_{0<|x|<1}|\bar{F}(y, x)|^{2} v(\mathrm{~d} x) \leq K\left(1+|y|^{2}\right), \forall y \in \mathbb{R} .
$$

Consequently, we have

$$
\begin{aligned}
& \int_{0}^{t} \int_{(0<|x|<1)}\left|\bar{F}\left(X_{s-}, x\right)\right|^{2} v(\mathrm{~d} x) \mathrm{d} s \\
& \quad \leq \int_{0}^{t} K\left(1+\left|X_{s-}\right|^{2}\right) \mathrm{d} s \leq K\left(1+\sup _{s \in[0, t]}\left|X_{s-}\right|^{2}\right) t,
\end{aligned}
$$

and hence, we get a.s.

$$
\int_{0}^{t} \int_{(0<|x|<1)}\left|\bar{F}\left(X_{s-}, x\right)\right|^{2} v(\mathrm{~d} x) \mathrm{d} s<\infty, \forall t \geq 0 .
$$

The online version of the original article can be found under https://doi.org/10.1007/s10959-015-0639-3.

Suprio Bhar

suprio@tifrbng.res.in; speedwlk@gmail.com

1 Tata Institute of Fundamental Research, Centre for Applicable Mathematics, Post Bag No 6503, GKVK Post Office, Sharada Nagar, Chikkabommsandra, Bangalore 560065, India 
The integrability condition (2) was originally stated as an assumption.

However, we now make a new integrability assumption. a.s.

$$
\int_{0}^{t} \int_{(0<|x|<1)}\left|\bar{F}\left(X_{s-}, x\right)\right|^{4} v(\mathrm{~d} x) \mathrm{d} s<\infty, \forall t \geq 0 .
$$

Note that, if $\bar{F}$ is bounded, using (2), (3) is satisfied.

Correction to Proof of Theorem 4.7 Equation (4.9) is to be read as

$$
\Delta X_{t}=\bar{F}\left(X_{t-}, \Delta N_{t}\right) \mathbb{1}_{\left(0<\left|\Delta N_{t}\right|<1\right)}+\bar{G}\left(X_{t-}, \Delta N_{t}\right) \mathbb{1}_{\left(\left|\Delta N_{t}\right| \geq 1\right)} .
$$

As such, the first observation mentioned in the article does not hold. However, due to (2), (3) and eq. (4.1), we still get

$$
\begin{aligned}
& \sum_{s \leq t}\left[\tau_{X_{s}} \phi-\tau_{X_{s-}} \phi+\Delta X_{s} \partial \tau_{X_{s-}} \phi\right] \\
& \quad=\int_{0}^{t} \int_{(0<|x|<1)}\left(\tau_{\bar{F}\left(X_{s-}, x\right)}-I d+\bar{F}\left(X_{s-}, x\right) \partial\right) \tau_{X_{s-}} \phi \tilde{N}(\mathrm{~d} s \mathrm{~d} x) \\
& \quad+\int_{0}^{t} \int_{(0<|x|<1)}\left(\tau_{\bar{F}\left(X_{s-}, x\right)}-I d+\bar{F}\left(X_{s-}, x\right) \partial\right) \tau_{X_{s-}} \phi v(\mathrm{~d} x) \mathrm{d} s \\
& \quad+\int_{0}^{t} \int_{(|x| \geq 1)}\left(\tau_{\bar{G}\left(X_{s-}, x\right)}-I d+\bar{G}\left(X_{s-}, x\right) \partial\right) \tau_{X_{s-}} \phi N(\mathrm{~d} s \mathrm{~d} x) .
\end{aligned}
$$

The proof now can be continued as before.

\section{References}

1. Applebaum, D.: Lévy Processes and Stochastic Calculus, 2nd edn. Cambridge Studies in Advanced Mathematics, vol. 116. Cambridge University Press, Cambridge (2009)

2. Bhar, S.: An Itō formula in the space of tempered distributions. J. Theoret. Probab. 30(2), 510-528 (2017) 